Mee Ling Tang, MBBS

Govindaraju Revadi, MS ${ }^{1}$

Raman Rajagopalan, MS ${ }^{2}$

Sushil Brito-Mutunayagam, MS

'Department of Otorhinolaryngology, Ampang Hospital, Selangor, Malaysia. 2Department of Otorhinolaryngology,

Faculty of Medicine, University of Malaya, Malaysia
Correspondence: Dr. Mee Ling Tang Department of Otorhinolaryngology, Ampang Hospital, Jalan Mewah Utara, Pandan Mewah, 68000 Ampang, Selangor Darul Ehsan.

Malaysia

Phone: +(601) 92596621

Telefax: +(603) 91023037

Email: meelingt2001@yahoo.com

Reprints will be available from the author.

The authors declared that this represents original materia that is not being considered for publication or has not been published or accepted for publication elsewhere in full or in part, in print or electronic media; that the manuscript has been read and approved by the authors, that the requirements for authorship have been met by the authors, and that the authors believe that the manuscript represents honest work.

Disclosures: The authors signed disclosures that there are no financial or other (including personal) relationships, intellectual passion, political or religious beliefs, and institutional affiliations that might lead to a conflict of interest.

\section{A Rare Case of Vertebrobasilar Dolichoectasia Presenting with Ipsilateral Facial Paresis and Concomitant Severe Sensorineural Hearing Loss}

\begin{abstract}
Objective: To report a case of vertebrobasilar dolichoectasia presenting with ipsilateral facial nerve paresis and concomitant severe sensorineural hearing loss.
\end{abstract}

Methods:

\author{
Design: Case Report \\ Setting: Secondary Government Hospital \\ Patient: One
}

Results: We report a case of vertebrobasilar dolichoectasia with concomitant ipsilateral facial nerve paresis and severe sensorineural hearing loss in an elderly female. She presented to us with left facial nerve palsy House-Brackmann Grade III and prior history of ipsilateral sensorineural hearing loss. MRI of the brain showed normal inner ear structures but revealed a dilated and tortuous basilar artery with compression on the left medulla and possible branches of anterior inferior cerebellar artery as it coursed superiorly and possible partial thrombosis of proximal basilar artery.

Conclusion: Concomitant facial nerve paresis and sensorineural hearing loss can be the clinical presentations of this rare but important condition. MRI is vital in diagnosing vertebrobasilar dolichoectasia.

Keywords: vertebrobasilar dolichoectasia, facial nerve palsy, sensorineural hearing loss, basilar artery

Vertebrobasilar dolichoectasia is also known as megadocholichoectasia, fusiform aneurysm of the vertebral and basilar arteries and tortuous vertebrobasilar system. Vertebrobasilar dolichoectasia is defined as an elongation and dilatation of the major arteries of the posterior fossa. ${ }^{1}$ It is a rare but well-known condition in clinical neurology with an incidence ranging from $0.06-5.8 \%{ }^{2}$

It is recognized as an important independent risk factor for stroke. ${ }^{3}$ Besides that, these dilated vessels can give rise to manifestations of interest to an ENT Surgeon. We report a case of vertebrobasilar dolichoectasia in an elderly female with an ipsilateral facial nerve paresis and concomitant sensorineural hearing loss. 
CASE REPORTS

\section{CASE REPORT}

A 73-year-old Malay woman with a past history of diabetes mellitus and hypertension presented to us with a sudden onset of incomplete left facial weakness for three days and a gradual left ear hearing impairment with intermittent tinnitus (sounds of wind) for four months. There was no history of otorrhoea or dizziness reported nor prior history of upper respiratory tract infection (URTI), head injury, vesicular rashes or stroke. She was on oral antihypertensives, subcutaneous insulin injection and aspirin for her medical illness.

On examination, she had left facial asymmetry with loss of left forehead groove and left nasolabial fold and full eye closure but weak to resistance consistent with House-Brackmann grading III. The otoscopic findings were normal. Tuning fork tests showed negative Rinne test on the left ear with Weber test lateralizing to the right, suggestive of left sensorineural hearing loss (SNHL). The rest of the cranial nerve examination was normal with negative cerebellar signs. No other neurological deficits were noted in both upper and lower limbs.

Tympanometry was Type A bilaterally and pure tone audiometry (PTA) showed left moderate to severe sensorineural hearing loss with right ear normal hearing with an $8 \mathrm{kHz}$ dip.

She completed a course of oral steroids and neurobion and underwent facial physiotherapy.

The facial weakness resolved completely after a few months with persistent asymmetry of the left-sided severe sensorineural hearing loss. A contrast-enhanced Magnetic Resonance Imaging of the Internal Acoustic Meatus revealed a tortuous, elongated and ectatic basilar artery, measuring $4.1 \mathrm{~mm}$ in its widest axial diameter. There was compression on the left medulla and possibly branches of the anterior inferior cerebellar artery as it coursed superiorly. The left vestibulocochlear nerve did not appear directly compressed. There was mixed heterogeneity of intraluminal signal intensity suspicious of thrombosis. There was no abnormal increase in signal intensity within the pons to suggest infarcts. The cerebellopontine angle, internal acoustic meatus, and all the inner, middle and outer ear structures were reported as normal.

She was diagnosed to have atherosclerotic vertebrobasilar dolichoectasia with partial thrombosis of the proximal basilar artery.

\section{DISCUSSION}

Vertebrobasilar dolichoectasia is an anatomic variant consisting of enlargement and dilatation of the vertebrobasilar artery which is often tortuous and elongated. ${ }^{1}$ Histologically, there are early fragmentation of the internal elastic lamina, intimal hyperplasia and intramural hemorrhage in these dilated arteries.

This condition is most likely due to prolonged systemic arterial hypertension. ${ }^{4}$ As a result of the increased diameter of the dilated artery, there is presence of both orthograde and retrograde flow within the same arterial segment. This can create turbulence in the blood flow of the artery causing thrombus formation. ${ }^{2}$

There are various clinical presentations of vertebrobasilar dolichoectasia depending on the location and nature of the vessel. $M$ Titlic et al. performed a topical review on its various clinical manifestations. ${ }^{2}$ The vicinity of the ectatic basilar artery in the brainstem can cause compression symptoms of the surrounding structures of the brainstem leading to cranial nerve palsies. Cranial nerves palsies involving IVth (trochlear) nerve, VIth (abducens) nerve, VIIth (facial) nerve, VIllth (vestibulocochlear) nerve and even Vth (trigeminal) nerve and optic tract, albeit rare have all been described. The presentation includes diplopia, trigeminal neuralgia, hemifacial spasm and sensorineural hearing loss with vertigo. The cranial nerves compressed may be isolated or combined depending on the diameter of the basilar artery. ${ }^{1}$ To the best of our knowledge, no case has been described in the English literature to involve both VIIth (facial) and VIIIth (vestibulocochlear) nerves together.

Due to the dilated nature of the artery, the turbulence in blood flow can also cause vertigo and pulsating tinnitus in the affected patient. ${ }^{2}$ These symptoms can be very debilitating.

Facial nerve paresis as a sole presenting complaint in vertebrobasilar dolichoectasia is rare as there is only one case so far reported by Mishra et al. regarding recurrent incomplete alternating facial hemiparesis secondary to a dolichoectatic vertebrobasilar artery in a middle-aged man. In that reported case, the man eventually required an operation and clipping was done. Postoperatively, there was improvement in the facial weakness up to $95 \%$ over a 5 -month duration. ${ }^{5}$ In our case study, the patient presented with ipsilateral facial nerve paresis which coincided with the compressed side of the brainstem. However, there was no demonstration of direct compression of the facial nerve. We speculate that the facial nerve paresis in this patient was the consequence of either compression of the arterial supply to the facial nerve or a thrombus formation which later recanalized. The facial nerve receives an arterial supply from branches of the anterior inferior cerebellar artery between its exit from the pons and internal auditory meatus. ${ }^{6}$ Microembolism of the thrombi is a possibility in view of the suspicious thrombus seen on MRI. However, an idiopathic cause or Bell's palsy also cannot be ruled out in her case.

The usual etiologies of unilateral sensorineural hearing loss include noise-induced, ototoxic, basal meningitic, acoustic neuroma and vascular causes. Vascular causes of sensorineural hearing loss are usually due to embolism or thrombosis of the labyrinthine or cochlear artery. In our case, the persistent unilateral sensorineural hearing loss prompted us to investigate for a possible retrocochlear cause and this rare condition was an incidental finding. The unilateral sensorineural hearing loss may be attributed to the affected arterial supply from the labyrinthine artery that supplies the inner ear. In the reported cases, patients with sensorineural hearing loss secondary to vertebrobasilar dolichoectasia may or may not have vestibular component involvement, 
and their outcomes vary from temporary to residual or permanent total hearing loss. ${ }^{5,7-9}$

Tinnitus has been reported secondary to turbulent blood flow from the ectatic basilar artery but in her case, it may also possibly be part of the symptoms of sensorineural hearing loss.

Other than the clinical manifestations mentioned above, the initial presentation of vertebrobasilar dolichoectasia includes cerebellar dysfunction, central sleep apnea, arterial dissection or aneurysm, ischemic or hemorrhagic stroke and hydrocephalus. ${ }^{1,5}$

Magnetic resonance imaging (MRI) and magnetic resonance angiography (MRA) of the brain has been the method of choice in the study of vertebrobasilar dolichoectasia. ${ }^{10}$ MRI is non-invasive and can display vascular anatomy and its related posterior fossa structures, and delineate mural thrombi. MRA has the additional advantage of demonstrating the direction of vascular blood flow.

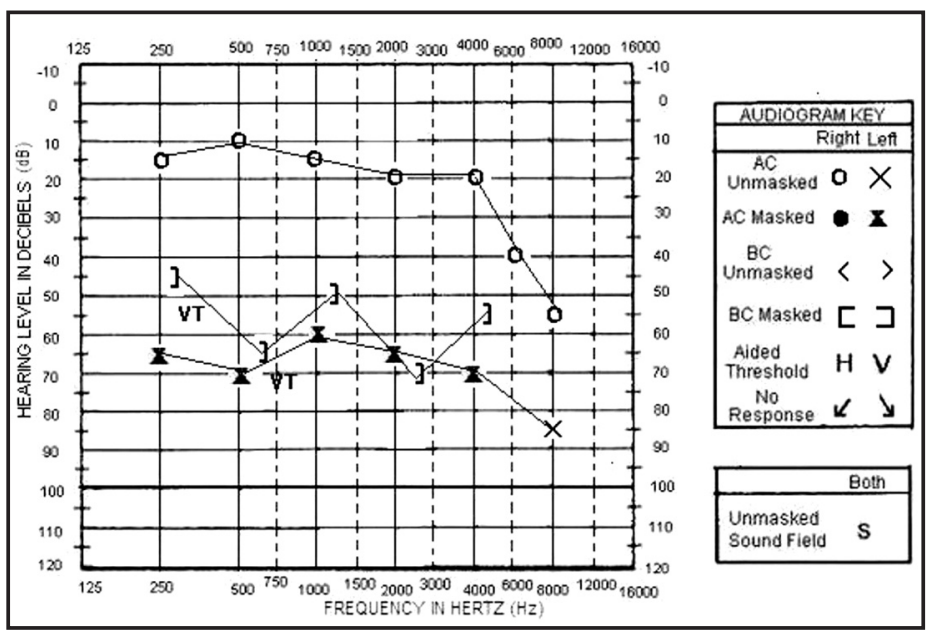

Figure 1. The Pure tone audiogram showed normal hearing with an $8 \mathrm{kHz}$ dip in the right ear and moderate to severe sensorineural hearing loss in the left ear.
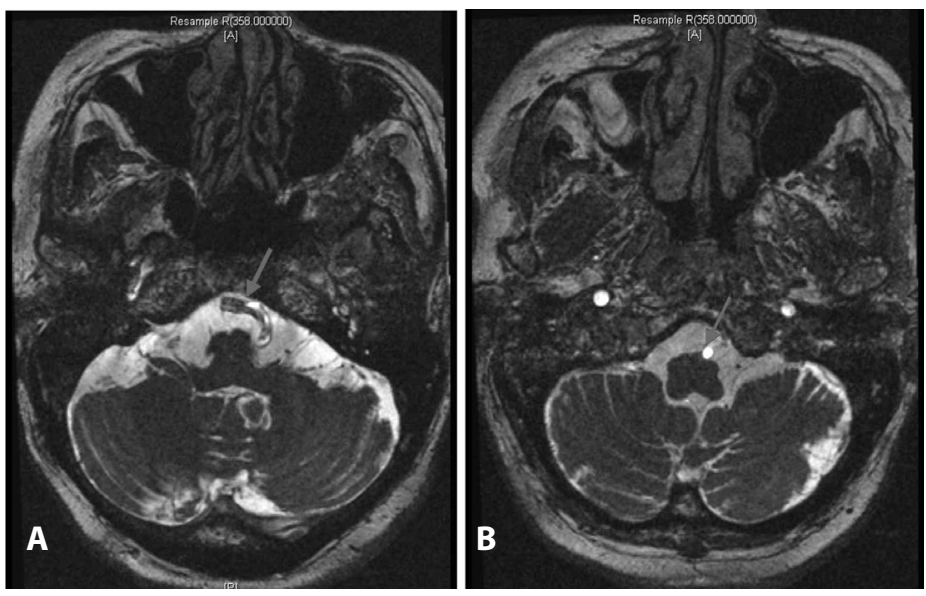

Figure 1. A. A cross-section T2 weighted MRI of the cerebellum showed a tortuous, elongated and ectatic basilar artery (arrow) measuring $4.1 \mathrm{~mm}$ in its widest axial diameter. Mixed heterogeneity of intraluminal signal is suspicious of thrombosis. B. A cross-section T2 weighted MRI of the cerebellum demonstrated compression of the contrasted basilar artery (arrow) on the left medulla.
There is a role for surgery for the treatment of cranial nerve disorders such as trigeminal neuralgia and hemifacial spasm. Microvascular decompression surgery was first introduced in the 1960s and popularized after 15 years. This surgery aims to alleviate the symptoms completely with minimal surgical complications. It is usually done by interposing Teflon, felt or other synthetic implants between the dilated vessel and the compressed nerves. ${ }^{4}$

We speculate that both the SNHL and facial nerve paresis could be a manifestation of VBD that occurred asynchronously. The benefit of surgery is not clear in this patient, however, it is important to educate her about the importance of antiplatelet treatment and emphasize the risk not only for stroke but recurrent facial hemiparesis/palsy as well as involvement of other cranial nerves reported in the literature. A cohort study by Ubogu et al. suggested that vertebrobasilar dolichoectasia is an independent risk factor for stroke. ${ }^{3} \mathrm{It}$ is also important to be aware of the progression of this condition which includes dissection, aneurysm, $\mathrm{SAH}$ and hydrocephalus.

\section{REFERENCES}

1. Smoker WR, Corbett JJ, Gentry LR, Keyes WD, Price MJ, McKusker S. High-resolution computed tomography of the basilar artery: 2. Vertebrobasilar dolichoectasia: clinical-pathological correlation and review. AJNR Am J Neuroradiol 1986 Jan-Feb; 7(1):61-72.

2. Titlic M, Tonkic A, Jukic I, Kolic K, Dolic K. Clinical manifestations of vertebrobasilar dolichoectasia. Bratis/ Lek Listy. 2008; 109(11): 528-530.

3. Ubogu EE, Zaidat $\mathrm{OO}$. Vertebrobasilar dolichoectasia diagnosed by magnetic resonance angiography and risk of stroke and death: a cohort study. J Neurol Neurosurg Psychiatry. 2004 Jan; 75(1):22-26.

4. Pereira-Filho A, Faria M, Bleil C, Kraemer JL. Brainstem compression syndrome caused by vertebrobasilar dolichoectasia: microvascular repositioning technique. Arq. Neuropsiquiatr. 2008 Jun;66(2b): 408-411.

5. Melo AA, Leão FS, Campos AJC, Antunes MRTA, Bunzen D, Neto SSC. Megadolicho basilar artery as a cause of asymmetrical sensorineural hearing loss - case report. Int. Arch. Otorhinolaryngol. 2011 Jul-Sep;15(3):385-387. DOI: 10.1590/S1809-48722011000300019.

6. Blunt MJ. The blood supply of the facial nerve. J Anat. 1954 Oct; 88(4): 520-526.

7. Nuti D, Passero S, Di Girolamo S. Bilateral Vestibular loss in vertebrobasilar dolichoectasia. $J$ Vestib Res. 1996 Mar-Apr; 6(2):85-91.

8. Büttner $\mathrm{U}$, Ott M, Helmchen C, Yousry $T$. Bilateral loss of eighth nerve function as the only clinical sign of vertebrobasilar dolichoectasia. J Vestib Res. 1995 Jan-Feb; 5(1):47-51.

9. Senkal HA, Özkan S, Oguz KK, Turan E. Dolichoectatic and tortuous vertebrobasillary arterial system causing progressive left-sided hearing loss in a patient with previous right-sided deafness. Mediterr J Otol 2008; 4: 143-147.

10. Vieco PT, Maurin EE 3rd, Gross CE. Vertebrobasilar dolichoectasia: evaluation with CT Angiography. AJNR Am J Neuroradiol. 1997 Aug; 18(7):1385-1388. 